\title{
Respostas de glicose, afeto básico e percepção subjetiva de esforço durante exercício resistido em mulheres com diabetes tipo 2
}

http://dx.doi.org/10.11606/1807-5509202000020215

\author{
Bruno Marcello Rodrigues dos SANTOS* \\ Loumaíra Carvalho da CRUZ, $Z^{* * * * * *}$ \\ Alfredo Anderson TEIXEIRA-ARAUJ O* \\ Thaise Camila Oliveira Gomes ROCHA* \\ Karoline Teixeira Passos de ANDRADE ${ }^{* * * *}$ \\ Sérgio Rodrigues MOREIRA*
}

*Universidade Federal

do Vale do São

Francisco, Petrolina, $P E$, Brasil.

**Centro Universitário Leão Sampaio Juazeiro do Norte, CE, Brasil.

***Departamento de Educação Física, Universidade Regional do Cariri, Iguatu, CE, Brasil.

**** Universidade de Pernambuco Petrolina, PE, Brasil.

O objetivo primário do estudo foi analisar as respostas de glicose (GLIC) e secundariamente o comportamento da percepção subjetiva de esforço (PSE) e domínio afetivo durante exercício resistido (ER) de diferentes intensidades em mulheres com diabetes mellitus tipo 2 (DM2). Oito mulheres com DM2 foram submetidas a sessões randomizadas, sendo: a) Dia 1 - Controle (CON40 ou CON80) e b) Dia 2 - ER (ER40 ou ER80), realizado em três circuitos de sete exercícios cada, durante um período de 40 minutos. Foram avaliados: GLIC, PSE, feeling scale (FS) e Felt Arousal Scale (FAS). A GLIC reduziu significativamente para ER40 $\left(140,0 \pm 19,1 \mathrm{mg} \cdot \mathrm{dL}^{-1}\right)$ quando comparado ao repouso $\left(228,3 \pm 16,6 \mathrm{mg}^{-\mathrm{dL}^{-1}}\right), \operatorname{CON} 40(241,3 \pm 53,2$ mg.dL $\left.{ }^{-1}\right), \operatorname{CON} 80\left(220,9 \pm 67,9 \mathrm{mg} \cdot \mathrm{dL}^{-1}\right)$ e ER80 $\left(212,2 \pm 70,9 \mathrm{mg} \cdot \mathrm{dL}^{-1}\right)$ na média geral da sessão $(\mathrm{P}<0,001)$ e a cada 5 minutos $(P<0,001)$. Para FS e FAS não ocorreu efeito principal do tempo e nem diferenças entre sessões $(P>0,05)$. Diferença significativa ocorreu na PSE entre sessões (ER40: 11,4 $\pm 1,2$ pts vs. ER80: 12,9 $\pm 1,5$ pts; $P<0,05)$. A sessão ER40 promoveu maior redução da GLIC e, apesar de diferir na PSE, ambas intensidades de ER mantiveram os resultados de FS e FAS.

Palavras-Chave: Esforço Físico; Diabetes Mellitus; Glicose; Percepção.

\section{Introdução}

O diabetes mellitus tipo 2 (DM2) é uma doença endócrina, proveniente de falhas nos mecanismos de ação da insulina (resistência à insulina) e desordens metabólicas, e que tem a hiperglicemia como sua principal característica e maior fator de propensão ao desenvolvimento de complicaçôes cardiovasculares ${ }^{1}$. O DM2 pode desencadear condiçóes clínicas adversas ao paciente, o que pode interferir na adoção de medidas terapêuticas ${ }^{2}$. Nesse sentido, a investigaçâo de abordagens que possam promover benefícios fisiológicos, sem gerar agravos perceptuais, deve ser considerada.

As terapias que visam atuar no tratamento do DM2 possuem seu foco voltado principalmente para o controle glicêmico e melhorias na capacidade de metabolização da glicose, uma vez que reduçôes na glicemia diminuem a instalaçáo e a progressão de complicaçôes vasculares $^{3-5}$. O paciente com DM2 sofre com a exposição diária a repetidas excursóes glicêmicas (picos hiperglicêmicos) que acontecem nas condiçóes pós-prandiais ${ }^{6}$. Como tais eventos podem exibir uma relaçáo ainda mais importante com o surgimento de comorbidades $^{1,7}$, se faz necessário investigar abordagens eficientes e direcionadas na redução da glicemia.

O exercício resistido (ER) tem se mostrado efetivo na redução da hiperglicemia em pessoas com $\mathrm{DM} 2^{8-10}$ e sua prática é atualmente recomendada pelas diretrizes do American College of Sports Medicine ${ }^{11}$ e pela American Diabetes Association ${ }^{3}$. Contudo, esta modalidade de exercício ainda necessita de mais evidências com relação à diferentes intensidades (leve vs. pesada) sobre as alterações nos valores de glicose plasmática durante a sua realização.

Por outro lado, sabe-se que o DM2 traz consigo uma importante demanda psicossocial interferindo na autonomia e autocuidado dos pacientes, o que aumenta 
a predisposição à incidência de quadros depressivos ${ }^{12}$. Essa condição pode influenciar negativamente o paciente com DM2 a adotar medidas terapêuticas para o tratamento da doença2. Nota-se que a atividade física, por exemplo, não tem se tornado uma prática comum entre a população com DM2 $2^{13-16}$.

Nesse sentido, o campo da psicobiologia tem investigado as respostas do afeto básico - sensaçóes associadas ao tônus hedônico (prazer-desprazer) e grau de ativação ${ }^{17}$ - que emergem durante o exercício físico, em busca de possíveis relações com a aderência a um estilo de vida fisicamente ativo. De modo geral, estes estudos têm demonstrado que, durante o exercício físico, o afeto básico se comporta de modo variável em resposta à intensidade do exercício físico ${ }^{18}$, e este afeto teria importante associação com a aderência de um

\section{Método}

\section{Amostra}

Dez mulheres com DM2 foram selecionadas para participar do estudo, sendo recrutadas com o auxílio de agentes comunitários de saúde e a partir de reunióes individuais e/ou coletivas em postos de saúde local, além da divulgação por meio de cartazes informativos em estabelecimentos públicos e privados diversos da cidade de Petrolina - PE. O estudo teve como critérios de inclusão: a) ser do sexo feminino, b) diagnosticada com DM2, c) estar clinicamente estável e, d) ter idade entre 40 e 60 anos. Os critérios de exclusão foram: a) fazer uso de insulina exógena, b) ter obesidade mórbida (IMC > $40 \mathrm{~kg} / \mathrm{m} 2$ ), c) apresentar glicose descompensada, d) apresentar anormalidade no ECG de repouso ${ }^{21}$, e) apresentar doença cardíaca, f) apresentar retinopatia diabética proliferativa, g) apresentar comportamento fisicamente ativo ${ }^{19}$.

Limitadas são as informações quanto às respostas afetivas ao esforço físico em pessoas com DM2. Kopp et al. ${ }^{20}$ verificaram que uma sessão de caminhada vigorosa (exercício aeróbio) é capaz de despertar influências positivas no afeto e bem-estar psicossocial de pessoas com DM2. No entanto, nenhum estudo verificou ainda o efeito de diferentes intensidades de ER sobre as respostas afetivas em mulheres com DM2, especialmente analisando em paralelo a resposta de glicose. Desta forma, o presente estudo teve como objetivo primário analisar as respostas de glicose e secundariamente verificar o comportamento da percepção subjetiva de esforço e domínio afetivo durante ER de diferentes intensidades em mulheres com DM2. neuropatia autonômica grave, h) apresentar qualquer amputação de membros, i) apresentar hipertensão arterial não controlada (sistólica > $160 \mathrm{mmHg}$ e/ou diastólica $>100 \mathrm{mmHg}$ ), j) apresentar nefropatia diabética (albuminúria $\geq 14 \mathrm{mg} / \mathrm{L}$ ou $>30 \mathrm{mg} / 24$ h), l) apresentar insuficiência renal crônica3,4, m) qualquer problema ósteo-mio-articular que limitasse a realização dos exercícios e, n) ser tabagista. Todas as participantes foram informadas dos riscos, benefícios e objetivos do estudo e assinaram um termo de consentimento livre e esclarecido. Durante o estudo, duas voluntárias apresentaram desistência na participação por motivos pessoais. Dessa forma, o número final de participantes na amostra investigada foi oito. As características gerais da amostra são apresentadas na TABELA 1. 
TABELA 1 - Média ( \pm DP) das características das participantes.

\begin{tabular}{ll} 
Características gerais & \\
$\mathrm{n}$ & 8 \\
Idade (anos) & $55,4 \pm 4,1$ \\
Massa corporal (kg) & $67,4 \pm 8,1$ \\
Estatura (cm) & $155,9 \pm 2,5$ \\
IMC (kg.m-2) & $27,8 \pm 3,7$ \\
Percentual de gordura (\%) & $24,2 \pm 4,2$ \\
NAF (min.sem-1) & $119,3 \pm 39,7$ \\
$\quad$ Sedentária & $0(0 \%)$ \\
$\quad$ Irregularmente Ativa B & $5(63 \%)$ \\
$\quad$ Irregularmente Ativa A & $0(0 \%)$ \\
$\quad$ Ativa & $3(38 \%)$ \\
$\quad$ Muito Ativa & $0(0 \%)$ \\
Diagnóstico DM2 (anos) & $4,8 \pm 3,9$ \\
Medicaçáo (n) & \\
Metformina 850mg & $5(62,5 \%)$ \\
Metformina 850mg + Glibenclamida 5mg & $1(12,5 \%)$ \\
Metformina 850mg + Glibenclamida 2,5mg & $1(12,5 \%)$ \\
Apenas dieta & $1(12,5 \%)$ \\
Teste de 1 $\boldsymbol{R} \boldsymbol{M}$ & \\
Supino sentado (kg) & $27,3 \pm 6,9$ \\
Extensora (kg) & $48,8 \pm 15,6$ \\
Voador (kg) & $20,0 \pm 6,4$ \\
Flexora (kg) & $46,5 \pm 13,1$ \\
Puxada frente (kg) & $33,0 \pm 8,8$ \\
Leg Press (kg) & $57,1 \pm 12,0$ \\
Remada sentada (kg) & $36,3,2$ \\
\hline
\end{tabular}

\section{Avaliação inicial}

As voluntárias foram submetidas a um exame de ECG de repouso, no qual apenas poderiam realizar o teste de uma repetição máxima (1RM) e as sessóes experimentais, após normalidade do laudo atestado pelo médico cardiologista. $\mathrm{Na}$ primeira visita, as participantes responderam anamnese sobre histórico de saúde com estratificação de risco ${ }^{11}$, nível de atividade física (NAF) e foram submetidas à avaliaçáo antropométrica com medidas de massa corporal, estatura, cálculo do índice de massa corporal e percentual de gordura através de equação preditiva utilizando as dobras cutâneas triciptal, subescapular e coxa medial ${ }^{22}$.

\section{Familiarização com os Procedimentos}

Uma familiarizaçáo com três dias alternados durante o período de duas semanas foi realizada com o protocolo de ER, em que as voluntárias foram orientadas na execuçáo correta dos exercícios, os quais foram na seguinte ordem: 1) supino na máquina, 2) cadeira extensora, 3) crucifixo na máquina, 4) cadeira flexora, 5) puxada alta frontal, 6) leg-press e 7) remada sentada. Ao longo da familiarização, as voluntárias receberam informaçôes sobre a utilização das escalas de afeto básico (FS - Feeling Scale), ativação (FAS - Felt Arousal Scale) e percepção subjetiva de esforço (PSE). 


\section{Teste de Uma Repetição Máxima (1RM)}

As voluntárias foram submetidas a um exame de ECG de repouso, no qual apenas poderiam realizar o teste de uma repetição máxima (1RM) e as sessóes experimentais, após normalidade do laudo atestado pelo médico cardiologista. Na primeira visita, as participantes responderam anamnese sobre histórico de saúde com estratificação de risco ${ }^{11}$, nível de atividade física (NAF) e foram submetidas à avaliação antropométrica com medidas de massa corporal, estatura, cálculo do índice de massa corporal e percentual de gordura através de equação preditiva utilizando as dobras cutâneas triciptal, subescapular e coxa medial ${ }^{22}$.

A força máxima foi avaliada através do teste de $1 \mathrm{RM}$, o qual fora realizado $48 \mathrm{~h}$ após a última sessão de familiarização nos exercícios citados anteriormente, seguindo a mesma ordem, com um máximo de quatro tentativas para cada $\mathrm{um}^{22}$. Foram seguidas as recomendaçóes de Kraemer e $\mathrm{FRY}^{23} \mathrm{com}$ a realização de um aquecimento com 10 a 15 repetiçóes e carga equivalente a 40 a $60 \%$ da força máxima percebida. Após o aquecimento, um intervalo de 2 minutos para recuperação foi realizado, enquanto a carga foi incrementada para a realizaçáo do teste. O movimento foi realizado com a voluntária podendo ou náo vencer a resistência oferecida e, após um intervalo de 3 a 5 minutos, a carga foi respectivamente, aumentada ou diminuída para permitir a realização de uma única repetição $0^{24}$.

\section{Dieta, Atividade Física e Medicação}

Durante a pesquisa as participantes receberam um desjejum padronizado contendo $285 \mathrm{kcal}$, sendo $45 \mathrm{~g}(180 \mathrm{kcal})$ de carboidratos, $6 \mathrm{~g}$ (24 kcal) de proteínas e $9 \mathrm{~g}(81 \mathrm{kcal})$ de gorduras.9 $\mathrm{O}$ desjejum foi administrado sempre no mesmo horário (7h da manhã). Quarenta e oito horas prévias às intervençóes, as participantes foram orientadas a não ingerir bebidas contendo álcool e/ou cafeína, bem como a se abster de qualquer exercício e/ou trabalho físico extenuante, com exceção dos exercícios propostos nos métodos do estudo. Ainda, as participantes foram orientadas a manterem suas rotinas normais de dieta, as quais foram recordadas para posterior cálculo da ingestão energética diária de alimentos, a qual não demonstrou diferenças significativas $(\mathrm{P}>0,05)$ entre as sessóes CON40, CON80, ER40 e ER80 (TABELA 2).
Das participantes, 7 faziam uso de medicação oral hipoglicemiante (Metformina $[n=5]$, Metformina em combinação com sulfonilureia [n=2]) e uma realizava apenas controle dietético (TABELA 1).

\section{Sistema de Monitorização Contínua da Glicose (SMCG)}

As medidas de glicose (GLIC) foram realizadas pelo SMCG através do equipamento Guardian REAL-Time (Medtronic Minimed, Inc., Northridge, CA, USA), o qual consiste em um sensor-transmissor inserido através de uma agulha no tecido subcutâneo abdominal utilizando um dispositivo (Sen-Seter, Medtronic Minimed, Inc., Northridge, CA, USA) e um monitor para leitura com sensor de frequência de rádio sem fio. Este tipo de monitoramento permite acessar os níveis de glicose intersticial de forma contínua e foi devidamente validado ${ }^{25-27}$.

O sensor de glicose (Sof-SensorTM, Medtronic Minimed, Inc., Northridge, CA, USA) foi instalado no dia anterior à primeira sessão, sendo inserido seguindo instruções do fabricante. As calibraçóes do SMCG foram realizadas mediante a utilização de um monitor de glicose capilar (AccuCheck Performa, Roche Diagnostics, Mannheim, Germany), sendo realizadas quatro vezes a cada período de $24 \mathrm{~h}$. As mensuraçóes de GLIC foram realizadas a cada 5 minutos durante 40 minutos das sessóes CON40, CON80, ER40 e ER80. Além das medidas realizadas pelo SMCG, também foram avaliadas a glicemia antes do desjejum às 7:00 da manhã (Glicose de jejum) e desjejum entre 8:00 e 8:20 da manhã (Glicose de repouso).

\section{PSE, FS e FAS}

Para aplicação das escalas perceptuais, foi realizada uma ancoragem de memória previamente com as voluntárias. A PSE foi avaliada utilizando uma escala a qual contém 15 pontos, onde o menor é representado pelo número 6 (sem esforço algum) e o ponto máximo representado pelo número 20 (esforço máximo), onde os números ímpares contêm pontos de ancoragem verbal ${ }^{28}$. A PSE foi avaliada durante os circuitos 1, 2 e 3 (C1, C2, C3) nas sessóes ER40 e ER80. A FS foi avaliada utilizando uma escala bipolar de 11 pontos, que varia de +5 (muito bom) a -5 (muito ruim) com descritores 
de ancoragem em todos os números ímpares, em que o 0 (zero) é o ponto neutro ${ }^{29}$.

A FAS foi avaliada utilizando uma escala de 6 pontos de identificação, com descritores de ancoragem nos pontos 1 (baixa ativação/ excitação) e 6 (alta ativação/excitação) ${ }^{30}$. Uma análise integrada interpolando as respostas de FS e FAS durante as sessôes experimentais foi realizada, visando obter o sentido o qual o domínio afetivo aponta a partir de diferentes quadrantes do modelo circumplexo (Alta ativação-Prazer; Alta ativação-Desprazer; Baixa ativação-Prazer; Baixa ativação-Desprazer) ${ }^{18}$.

Durante as sessóes de ER, todas as escalas foram apresentadas em ordem randomizada durante o repouso e ao final de cada um dos três circuitos (FIGURA 1).

\section{Sessões Experimentais}

As participantes com DM2 chegaram ao laboratório até às 6 h50 para serem submetidas a uma medida capilar de glicose no jejum. Após, às 7h00, o desjejum padronizado ( $285 \mathrm{kcal}$ ) foi administrado. Ao final da refeição, as voluntárias foram encaminhadas a uma sala de preparação, onde permaneceram sentadas até o momento de realização da sessão experimental. Às 8 h00 foi iniciado o momento repouso com duração de 20 minutos, em que nos últimos 5 minutos foi registrado o valor de glicose pós-prandial. Às 8h20, ao final do repouso, foi realizada apresentação das escalas para acessar as respostas afetivas (FS e FAS) de repouso. Em seguida foi iniciada a sessão controle $(\mathrm{CON})$ ou ER. A FIGURA 1 apresenta o desenho experimental do estudo.

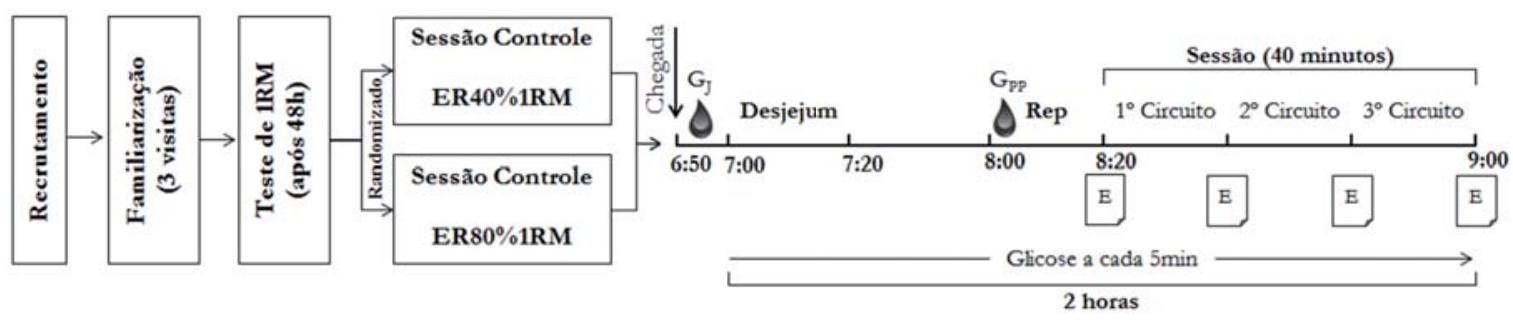

FIGURA 1 - Desenho experimental.

Sessão Controle: As sessóes controle do ER40 (CON40) e controle do ER80 (CON80) foram realizadas com as voluntárias permanecendo sentadas durante 40 minutos no mesmo ambiente de realização das sessóes de ER.

Exercício Resistido: Vinte quatro horas após a realização da sessão controle, foi realizada uma das sessôes de ER (ER40 ou ER80), com duração de 40 minutos, sendo compostas de 3 circuitos com 7 exercícios (supino na máquina, cadeira extensora, crucifixo na máquina, cadeira flexora, puxada alta frontal, leg-press e remada sentada). As intensidades do ER foram estabelecidas com base na classificação do $\mathrm{ACSM}^{11}$ e da $\mathrm{ADA}^{3}$ para indivíduos com DM2 $2^{15}$. Para as sessóes de ER40 e ER80 foram realizadas, respectivamente, 16 e 8 repetições em cada exercício com intervalo de recuperação de 60 segundos e 90 segundos entre os exercícios e 120 segundos entre os circuitos de ER. A duração da repetição em cada série de
ER foi 3 segundos, sendo 1 segundo na fase concêntrica e 2 segundos na fase excêntrica do movimento.

\section{Análise estatística}

Foi realizada estatística descritiva com valores de média e desvio padrão nas diferentes variáveis do estudo e distribuição de frequência percentual da medicação utilizada e o NAF na amostra. A normalidade da distribuição dos dados foi confirmada pelo Shapiro-Wilk test. ANOVA twoway para medidas repetidas foi aplicada para a análise intra e entre sessóes CON40, CON80, ER40 e ER80. Post-hoc de Bonferroni foi aplicado para identificar os pares de diferenças. Teste $t$ de Student comparou a PSE entre as sessóes ER40 e ER80. A significância foi definida no nível de confiança de $\mathrm{P}<0,05$. A análise estatística foi realizada utilizando o software SPSS v. 22.0. 


\section{Resultados}

Não houve diferença significativa nos valores da GLIC de repouso $\left[\mathrm{F}(3,21)=2,099 ; \mathrm{P}=0,131 ; \eta^{2}\right.$ $=0,231]$ entre CON40, CON80, ER40 e ER80 (FIGURAS 2A e 2B; TABELA 2). Por outro lado, para a média total da GLIC (FIGURA 2A) durante as sessões, ocorreu um efeito principal de tempo $[\mathrm{F}(1,7)=$ 11,$\left.417 ; \mathrm{P}<0,001 ; \eta^{2}=0,620\right]$ e interação tempo*sessão
$\left[\mathrm{F}(3,21)=9,239 ; \mathrm{P}<0,001 ; \eta^{2}=0,569\right]$, onde foram evidenciadas diferenças da sessão ER40 para as sessōes CON40, CON80 e ER80. Ao analisar a resposta de GLIC a cada 5 minutos (FIGURA 2B), também foi verificado efeito principal de tempo $[\mathrm{F}(8,56)=3,612 ; \mathrm{P}$ $\left.<0,01 ; \eta^{2}=0,340\right]$ e interação tempo*sessão $[\mathrm{F}(24,168)$ $\left.=7,978 ; \mathrm{P}<0,001 ; \eta^{2}=0,533\right]$.
* $P<0,001$ em relação ao Repouso da sessão ER40. † $P<0,001$ em relação às sessões CON40, CON80 e ER80. Glic: Glicose; C1: circuito 1; C2: circuito 2; C3: circuito 3
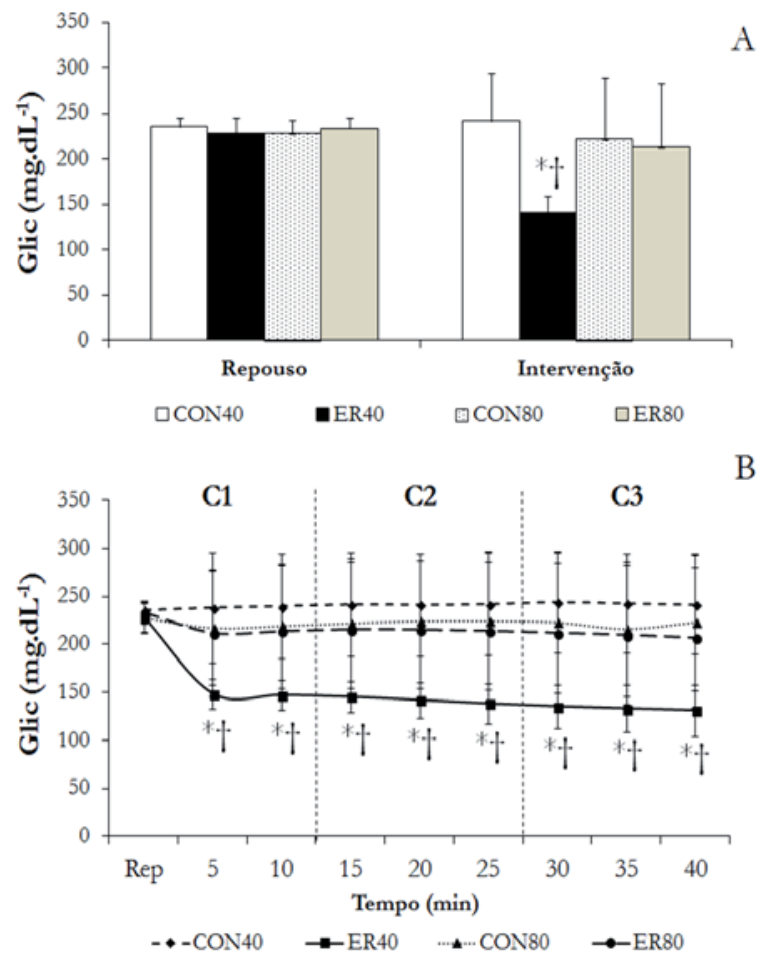

FIGURA 2 - Média ( \pm DP) da glicose durante as sessões experimentais (A) e glicose a cada 5 minutos (B).

A FS e FAS não sofreram efeito principal de tempo $\left[\mathrm{F}(3,21)=0,165 ; \mathrm{P}=0,919 ; \eta^{2}=0,023\right.$ e $\mathrm{F}(3,21)=$ 0,$712 ; \mathrm{P}=0,555 ; \eta^{2}=0,092$, respectivamente] e nem interação tempo*sessão $[\mathrm{F}(9,63)=1,131 ; \mathrm{P}=0,249$; $\eta^{2}=0,158$ e $\mathrm{F}(9,63)=1,653 ; \mathrm{P}=0,120 ; \eta^{2}=0,191$, respectivamente].

A FIGURA 3 apresenta a dinâmica temporal da resposta afetiva (FS e FAS) para as sessóes CON40,
ER40, CON80 e ER80. Embora ocorra a manutenção de todas as sessóes no quadrante de alta ativaçáo-prazer ao final da realização dos circuitos, é possível visualizar uma variaçăo temporal crescente, especialmente ao longo da execuçáo dos circuitos de ER.

A FIGURA 4 apresenta a resposta integrada de PSE entre as sessóes de ER40 e ER80, a qual foi estatisticamente diferente $[\mathrm{t}(7)=-1,776 ; \mathrm{P}=0,05]$. 


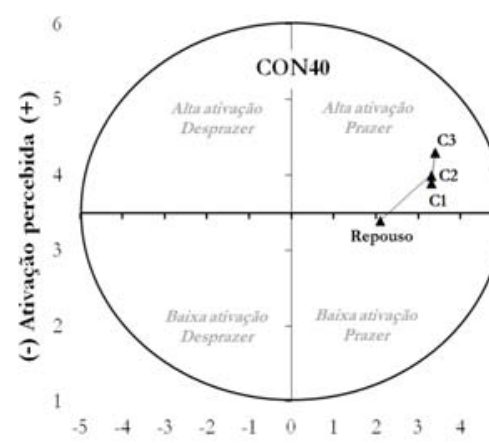

$(-)$ Valência afetiva $(+)$

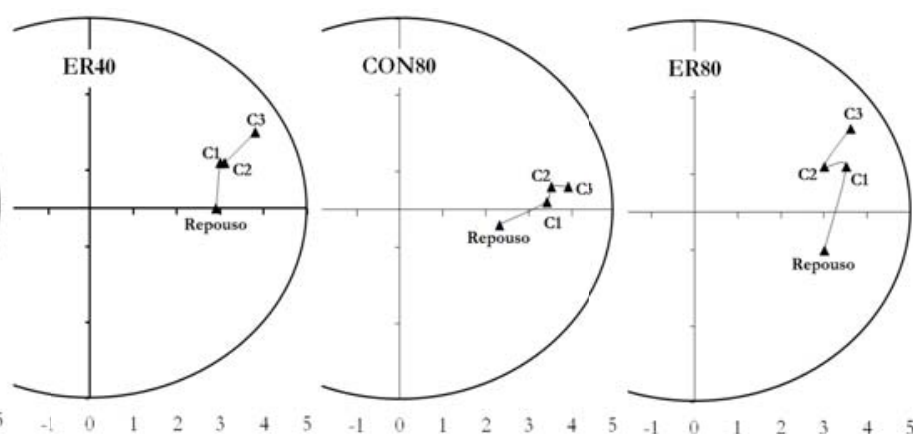

$(-)$ Valência afetiva $(+)$

(-) Valência afetiva (+)

(-) Valência afetiva (+)

FIGURA 3 - Modelo Circumplexo de análise bidimensional do afeto básico, realizado mediante a interpolação dos valores obtidos na feeling scale (FS) e felt arousal scale (FAS).

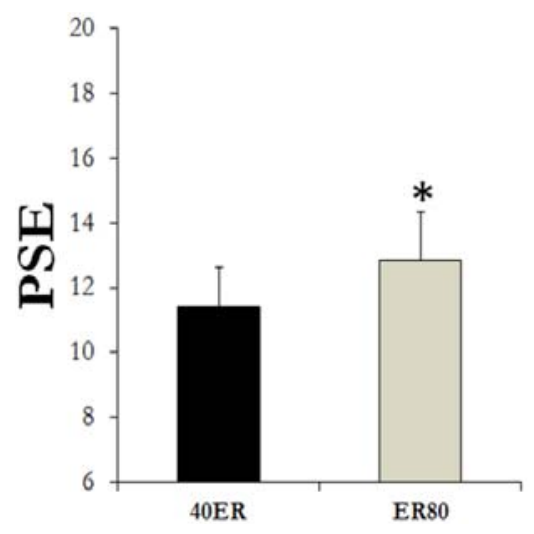

* $P<0,05$ em relação à sessão ER40.

FIGURA 4 - Média (†DP) da percepção subjetiva de esforço (PSE) durante as sessões ER40 e ER80.

\section{Discussão}

Os resultados demonstram que durante o ER de menor intensidade (ER40) ocorreu maior redução da GLIC quando comparado às sessóes controle sem exercício, mas especialmente quando comparado à sessão mais intensa de ER (ER80). Além disso, houve diferença significativa da sessão ER40 para o seu respectivo repouso tanto na média geral da GLIC, quanto durante os momentos a cada 5 minutos de mensuração na sessão (FIGURA 2).

Esses achados corroboram aos resultados obtidos por Moreira et al. ${ }^{9}$, os quais evidenciaram que durante o ER em muito baixa (23\% de $1 \mathrm{RM}$ ) e baixa ( $43 \%$ de $1 \mathrm{RM}$ ) intensidades, ocorre uma redução significativa dos valores de GLIC em comparação ao repouso das respectivas sessões e a sessão controle sem exercício. Esses resultados demonstraram que a sessão menos intensa foi mais efetiva em reduzir a área abaixo da curva de GLIC quando comparada a sessão mais intensa de ER $(\mathrm{P}<$ $0,05)$. O exercício físico realizado em alta intensidade tem sido associado ao aumento da atividade nervosa simpática e acentuada liberação de catecolaminas e glucagon, o que, por conseguinte potencializa o processo de gliconeogênese e glicogenólise hepática e muscular ${ }^{31-33}$. Dessa forma, especula-se que a açáo muscular intensa promovida na sessão ER80, tenha possibilitado uma maior produção de GLIC endógena 
quando comparado à sessão ER40.

A manutenção da hiperglicemia está associada com a redução do micro RNA tipo 1 , o qual fisiologicamente pode desencadear na elevação da endotelina e fibronectina no DM2, com consequente apoptose e danos em órgáos alvos e tecidos como rins, coração e retina ${ }^{34}$. Do ponto de vista clínico, os resultados encontrados no presente estudo sugerem a menor intensidade de exercício físico (ER40) como uma estratégia importante no controle glicêmico pósprandial de pacientes com DM2.

Em relação às respostas perceptuais, tem sido destacada na literatura uma relação inversa entre intensidade do exercício físico e valência afetiva. 18 Maiores intensidades de exercício físico resultam em um aumento da sensação de desprazer ou mesmo diminuição do prazer $^{35}$. No presente estudo não foram evidenciadas diferenças significativas da FS entre as sessōes de ER, as quais resultaram em estado prazeroso do início ao fim das sessóes. Uma das explicaçôes para determinado resultado pode ser devido à equalização do volume de trabalho realizado nas sessōes de ER. Como o objetivo foi verificar apenas o efeito de ambas as intensidades (ER40 e ER80), o estudo manteve o mesmo volume de trabalho realizado, sendo na sessão ER80 oito repetiçóes por série, enquanto na sessão ER40 foram 16 repetiçóes por série. Esses resultados sugerem que independente da intensidade, se a estruturação das variáveis dentro da sessão de ER (quantidade de repetiçóes, séries, tempo de recuperação) seguir princípios biológicos adequados ao treinamento, possíveis reduções do afeto básico poderão ser minimizadas, o que em longo prazo, quando em condiçôes compensadas de GLIC, poderia também contribuir na aderência do indivíduo com DM2 a um programa de treinamento mais intenso ${ }^{18,19}$.

A análise bidimensional das respostas perceptuais (FS e FAS) demonstrou uma manutenção do afeto básico em um estado prazeroso e com alta ativação (quadrante 'Alta ativação-Prazer') durante as sessóes experimentais (FIGURA 3). Sendo assim, sugere-se que, especialmente nas sessóes de ER40 e ER80, ocorreram características afetivas de "entusiasmo e excitaçáo" ao longo da realização do esforço ${ }^{17}$.

Ademais, a PSE foi sensível às intensidades de exercício aplicadas no presente estudo (FIGURA 4), uma vez que a sessão ER80 ocorreu na média de $13,0 \pm 1,5$ pontos, classificando-a segundo $\mathrm{BoRG}^{28}$ como 'Um pouco difícil' em comparação à sessão ER40 $(11,0 \pm 1,0$ pontos - 'Leve'). Esses achados corroboram com a literatura em que a PSE se relaciona de forma linear com a intensidade do exercício de força ${ }^{36,37,38}$.

Uma principal limitação da presente investigação é o tamanho da amostra de mulheres com DM2. Contudo, mesmo com o número amostral reduzido a estatística ainda foi capaz de capturar significância entre as sessôes, o que justifica o potencial dos resultados apresentados. Outro aspecto a se considerar seria o período de uma hora pós-prandial para a realização das sessões de exercício (ER40 e ER80), o qual pode gerar questionamentos em relação aos resultados obtidos. Entretanto, o design randomizado cruzado do presente estudo minimiza essa limitação, em conjunto ainda aos procedimentos de ingestão alimentar, exercício físico e tempo os quais foram padronizados em todas as sessóes realizadas. Em adicional, outros trabalhos ${ }^{39,40}$ associados aos resultados do presente estudo, suportam a segurança fisiológica da metodologia empregada, no tocante a não ocorrência de possíveis eventos hipoglicemiantes durante e após a realização do exercício físico.

Conclui-se que o ER realizado em baixa intensidade (ER40) foi efetivo na redução dos valores de GLIC durante a realização da sessão, além de apresentar uma menor PSE em comparação à sessão de alta intensidade (ER80). Em relação ao afeto básico, independente da intensidade, o ER se mostrou prazeroso para mulheres com DM2.

\section{Agradecimentos}

Os autores agradecem à Coordenação de Aperfeiçoamento de Pessoal de Nível Superior - CAPES e ao Conselho Nacional de Desenvolvimento Científico e Tecnológico - CNPq (proc. 470593/2013-0).

\section{Conflito de interesses}

Os autores declaram não haver conflito de interesses ao presente estudo. 


\begin{abstract}
Glucose responses, basic affect and rating of perceived exertion during resistance exercise in woman with type 2 diabetes

The primary aim of the study was to analyze the glucose responses (GLUC) and secondarily the rate perceived exertion (RPE) and affective domain behavior during resistance exercise (RE) at different intensities in women with type 2 diabetes mellitus (T2DM). Eight women with T2DM were subjected to randomized sessions, being: a) Day 1 - Control (CON40 or CON80) and b) Day 2 - RE (RE40 or RE80), performed in three circuits of seven exercises each during a period of 40 minutes. Were evaluated: GLUC, RPE, feeling scale (FS) and Felt Arousal Scale (FAS). The GLUC significantly reduced in session RE40 (140.0 $\left.\pm 19.1 \mathrm{mg}^{-\mathrm{dL}^{-1}}\right)$ when compared resting condition $\left(228.3 \pm 16.6 \mathrm{mg}_{\mathrm{dL}}{ }^{-1}\right), \mathrm{CON} 40\left(241.3 \pm 53.2 \mathrm{mg} \cdot \mathrm{dL}^{-1}\right), \mathrm{CON} 80\left(220.9 \pm 67.9 \mathrm{mg} \cdot \mathrm{dL}^{-1}\right)$ and RE80 $\left(212.2 \pm 70.9 \mathrm{mg}^{-\mathrm{dL}^{-1}}\right)$ in the overall average session $(P<0.001)$ and every 5 minutes $(P<0.001)$. To FS and FAS there was no main time effect and no difference among sessions $(P>0.05)$. Significance difference occurred between sessions to RPE (RE40: $11.4 \pm 1.2$ pts vs. RE80: $12.9 \pm 1.5$ pts; $P<0.05$ ). RE40 session promoted greater reduction GLUC and, despite differing in RPE, both RE intensities maintained the results of FS and FAS.
\end{abstract}

KeYwords: Physical Exertion; Diabetes Mellitus; Glucose; Perception.

\title{
Referências
}

1. Ceriello A. Postprandial hyperglycemia and diabetes complications: Is It time to treat? Diabetes. 2004;54:1-7.

2. Rubin RR, Peyrot M. Psychological issues and treatments for people with diabetes. J Clin Psychol. 2001;57:457-78.

3. ADA. American Diabetes Association. Diagnosis and classification of diabetes mellitus. Diabetes Care. 2015;37:S81-S90.

4. SBD - Sociedade Brasileira de Diabetes - Diretrizes da Sociedade Brasileira de Diabetes: 2013-2014, 2015.

5. Stratton IM. Association of glycaemia with macrovascular and microvascular complications of type 2 diabetes (UKPDS 35): prospective observational study. Br Med J. 2000;321:405-12.

6. van Dijk JW, Manders RJ, Hartgens F, Stehouwer CD, Praet SF, van Loon LJ. Postprandial hyperglycemia is highly prevalent throughout the day in type 2 diabetes patients. Diabetes Res Clin Pract. 2011;93:31-7.

7. Ceriello A. Point: Postprandial glucose levels are a clinically important treatment target. Diabetes Care. 2010;33:1905-7.

8. Bacchi E, Negri C, Trombetta M, Zanolin ME, Lanza M, Bonora E, Moghetti P. Differences in the acute effects of aerobic and resistance exercise in subjects with type 2 diabetes: Results from the RAED2 Randomized Trial. PlosOne. 2012;7:e49937.

9. Moreira SR, Simóes GC, Moraes JF, Motta DF, Campbell CS, Simôes HG. Blood glucose control for individuals with type-2 diabetes. J Strength Cond Res. 2012;26:2806-11.

10. van Dijk JW, Manders RJ, Tummers K, et al. Both resistance- and endurance-type exercise reduce the prevalence of hyperglycaemia in individuals with impaired glucose tolerance and in insulin treated and non-insulin-treated type 2 diabetic patients. Diabetologia. 2012;55:1273-82.

11. ACSM. American College of Sports Medicine's Guidelines for exercise testing and prescription. 5th ed. Baltimore, Md: Willians \& Wilkins; 1995, p. 269-87.

12. Anderson RJ, Freedland KE, Clouse RE, Lustman PJ. The prevalence of comorbid depression in adults with diabetes: A meta-analysis. Diabetes Care. 2001;24:1069-78.

13. Asano RY, Gargaglione EML, Cruz LFR, et al. Fatores que influenciam a adesão de diabéticos à prática de exercícios físicos. Rev Bras Cienc Mov. 2015;23:5-11.

14. Boas LCGV, Foss MC, Foss-Freitas MC, Torres HC, Monteiro LZ, Pace AE. Adesão à dieta e ao exercício físico das pessoas com diabetes mellitus. Texto Contexto - Enferm. 2011;20:272-9.

15. Colberg SR, Sigal RJ, Fernhall B, et al. Exercise and type 2 diabetes: The American College of Sports Medicine and the American Diabetes Association. Diabetes Care. 2010;33:2692-6.

16. Faria HTG, Rodrigues FFL, Zanetti ML, Araújo MFM, Damasceno MMC. Fatores associados à adesão ao tratamento 
de pacientes com diabetes mellitus. Acta Paul Enferm. 2013;26:231-7.

17. Barrett LF, Bliss-Moreau E. Affect as a psychological primitive. Adv Exp Social Psychol. 2009;41:167-218.

18. Ekkekakis P, Parfitt G, Petruzzello SJ. The Pleasure and Displeasure People Feel When they Exercise at Different Intensities. Sports Med. 2011;41:641-71.

19. Williams DM, Dunsiger S, Ciccolo JT, Lewis BA, Albrecht AE, Marcus BH. Acute affective response to a moderate-intensity exercise stimulus predicts physical activity participation 6 and 12 months later. Psychol Sport Exerc. 2008;9:231-45.

20. Kopp M, Steinlechner M, Ruedl G, Ledochowski L, Rumpold G, Taylor AH. Acute effects of brisk walking on affect and psychological well-being in individuals with type 2 diabetes. Diabetes Res Clin Pract. 2012;95:25-9.

21. Chalela WA, Moffa PJ. Teste ergométrico. In: Negrão CE, Barreto ACP, Organizers. Cardiologia do Exercício: do atleta ao cardiopata. 3aed. Barueri: Editora Manole; 2010, p. 249-96.

22. Marins JCB, Giannichi RS. Avaliação e prescrição de atividade física: Guia prático. Rio de Janeiro: Shape, 1996.

23. Kraemer WJ, Fry AC. Strength testing: Development and evaluation of methodology. In: Maud PJ, Foster C (Eds.), Physiological assessment of human fitness; Champaign, IL: Human Kinetics; 1995, p. 115-38

24. Dias RMR, Cyrino ES, Salvador EP, et al. Influência do processo de familiarização para avaliação da força muscular em testes de 1-RM. Rev Bras Med Esporte. 2005;11:34-8.

25. Meade LT. The use of continuous glucose monitoring in patients with type 2 diabetes. Diabetes Technol Ther. 2012; 14:190-5.

26. Terada T, Loehr S, Guigard E, et al. Test-retest reliability of a continuous glucose monitoring system in individuals with type 2 diabetes. Diabetes Technol Ther. 2014;16:491-8.

27. Tavris DR, Shoaibi A. The public health impact of the minimed continuous glucose monitoring system $\left(\mathrm{CGMS}^{\oplus}\right)$ An assessment of the literature. Diabetes Technol Ther. 2004;6:518-22.

28. Borg GAV. Psychophysical bases of perceived exertion. Med Sci Sports Exerc. 1982;14:377-81.

29. Hardy CJ, Rejeski WJ. Not what, but how one feels: The measurement of affect during exercise. J Sport Exerc Psychol. 1989;11:304-17.

30. Svebak S, Murgatroyd S. Metamotivational dominance: A multimethod validation of reversal theory constructs. J Pers Soc Psychol. 1985;48:107-16.

31. Coker RH, KjaerM. Glucoregulation during exercise. Sports Med. 2005;35:575-83.

32. Marliss EB, Vranic M. Intense exercise has unique effects on both insulin release and its roles in glucoregulation: implications for diabetes. Diabetes. 2002;51:271-83.

33. Wasserman DH, Kang L, Ayala JE, Fueger PT, Lee-Young RS. The physiological regulation of glucose flux into muscle in vivo. J Exp Biol. 2011;214:254-62.

34. Feng B, Cao Y, Chen S, Ruiz M, Chakrabarti S. Reprint of: miRNA-1 regulates endothelin-1 in diabetes. Life Sciences. 2014; 118:275-280.

35. Ekkekakis P, Hall E, Petruzzello SJ. Variation and homogeneity in affective responses to physical activity of varying intensities: An alternative perspective on dose - response based on evolutionary considerations. J Sports Sci. 2005;23:477-500.

36. Costa A, Fernandes C. Utilização da percepção subjetiva do esforço para monitorização da intensidade do treino de força em idosos. Motricidade. 2007;3:37-46.

37. Tiggemann CL, Pinto RS, Kruel LFM. A Percepção de esforço no treinamento de força. Rev Bras Med Esporte. 2010;16:301-9.

38. Gearhart RF Jr, Goss FL, Lagally KM, et al. Ratings of perceived exertion in active muscle during high-intensity and low-intensity resistance exercise. J Strength Cond Res. 2002;16:87-91.

39. Colberg SR, Zarrabi L, Bennington L, et al. Postprandial walking is better for lowering the glycemic effect of dinner than pre-dinner exercise in type 2 diabetic individuals. J Am Med Dir Assoc. 2009;10(6):394-397.

40. Colberg SR, Grieco GR, Somma T. Exercise effects on postprandial glycemia, mood, and sympathovagal balance in type 2 diabetes. JAMDA. 2014;15:261e266. 

andersonaraujoba@gmail.com 